\title{
On the effect of pruning on the singularity structure of zeta functions
}

\author{
Per Dahlqvist \\ Mechanics Department \\ Royal Institute of Technology, S-100 44 Stockholm, Sweden
}

\begin{abstract}
We investigate the topological zeta function for unimodal maps in general and dynamical zeta functions for the tent map in particular. For the generic situation, when the kneading sequence is aperiodic, it is shown that the zeta functions have a natural boundary along its radius of convergence, beyond which the function lacks analytic continuation. We make a detailed study of the function $\prod_{n=0}^{\infty}(1-$ $z^{2^{n}}$ ) associated with sequences of period doublings. It is demonstrated that this function has a dense set of poles and zeros on the unit circle, exhibiting a rich number theoretical structure.
\end{abstract}

\section{Introduction and preliminaries}

In chaotic dynamics one aims at a global description of the phase space flow rather than a description of individual trajectories. The appropriate tool for this analysis is evolution operators (Ruelle-Perron-Frobenius operators). It requires a rather extensive mathematical apparatus to understand the spectrum of this operator in detail. The problem is that one has to restrict the functions space, acted on by the operator, in order to capture only the physically relevant spectrum. However it seems as if, in many cases, this relevant spectrum can be identified with the spectrum of poles of zeta functions or (even better) zeros of Fredholm determinants. These functions require only knowledge of the periodic orbits and their invariants, such as stability and length and no reference to specific function classes. The link between the spectra of evolution operator and their zeta functions has been rigorously established only for restricted classes of systems, like e.g. expanding (or hyperbolic) systems with a symbolic dynamics of finite subshift type [1, 2]. For such systems the Fredholm determinants may even be entire functions.

However, there are many ergodic systems of physical relevance that do not fall into this class of systems. We can then no longer expect singularities to be absent from the zeta function. There are strong indications that for intermittent systems the zeta functions exhibit branch point singularities 3, 4, 5. The corresponding branch cut could possibly be associated with a continous component of the spectrum of the evolution operator. There is evidence that these singularities 
do carry important information about the dynamics of the system, such as power law decay of correlations and phase transitions among e.g. the generalized Lyapunov exponents [4, 6, 7].

In this paper we will study the analytic structure of the zeta function for expanding systems with no symbolic dynamics of finite subshift type.

We will study the simplest conceivable system, namely one-dimensional unimodal (onehumped) maps $x \mapsto f_{\lambda}(x)=\lambda \cdot g(x)$ with one external control parameter $\lambda[8,9,10]$.

The kneading sequence $\underline{I}_{\lambda}$ is the orbit of the critical point, represented by a string of $R$ and $L$ 's depending on the branch of the map visited. The ordering of kneading sequences with respect to the parameter $\lambda$ do not depend on the details of the unimodal $g(x)$ if it has strictly negative Schwartzian derivative 10,8 . For the tent map this derivative is zero and the sequences of period doublings are squeezed into a zero length intervals in parameter space.

The kneading sequence can be of one of three types

a) It maps to the critical point again, after $n$ iterations. If so, we adopt the convention to terminate the kneading sequence with a $C$, and refer to the kneading sequence as finite.

b) Preperiodic, i.e. it is infinite but with a periodic tail.

c) Aperiodic. 11

Much dynamic information is encoded in the following one-parameter family of zeta functions

$$
1 / \zeta(\beta, z)=\prod_{p}\left(1-\frac{z^{n_{p}}}{\left|\Lambda_{p}\right|^{1-\beta}}\right) .
$$

The product in (11) runs over all primitive periodic orbits $p$, having period $n_{p}$ and stability $\Lambda_{p}=\left.\frac{d f^{n} p}{d x}\right|_{x=x_{p}}$ with $x_{p}$ being any point along $p$. We will only study a very special case of this function in detail, namely the topological zeta function

$$
1 / \zeta_{\text {top }}(z) \equiv 1 / \zeta(\beta=1, z)=\prod_{p}\left(1-z^{n_{p}}\right),
$$

and then turn to some speculations about $\beta<1$ at the end. The leading zero $z_{0}$ of $1 / \zeta_{\text {top }}(z)$ (the one with smallest modulus) and the topological entropy $h$ are related by $h=-\log z_{0}$. There is one unimodal map for which $1 / \zeta_{\text {top }}(z)$ contains all information concerning $1 / \zeta(\beta, z)$ for all $\beta$, namely the following piecewise linear map, called the tent map [12, 13, 14

$$
x \mapsto f(x)=\left\{\begin{array}{ll}
\lambda \cdot x & x \in[0,1 / 2] \\
\lambda \cdot(1-x) & x \in(1 / 2,1]
\end{array} .\right.
$$

where the parameter $\lambda \in[0,2]$ Since $\left|\Lambda_{p}\right|=\lambda^{n_{p}}$ we have

$$
1 / \zeta(\beta, z)=1 / \zeta_{\text {top }}\left(z / \lambda^{1-\beta}\right)
$$

The topological entropy is $h=-\log \lambda$.

The set of periodic points of the tent map is countable. A consequence of this fact is that the set of parameter values for which the kneading sequence is finite or eventually periodic are countable and thus of measure zero and consequently the kneading sequence is aperiodic for almost all $\lambda$. For general unimodal maps the corresponding statement is that the kneading sequence is aperiodic for almost all topological entropies.

It is, in fact, easy to write down the expanded topological zeta function corresponding to a given kneading sequence $\underline{I}_{\lambda}=P C$, of length $n$, where $P=s_{1} s_{2} \ldots s_{n-1}$ is a string of symbols where each symbol $s_{i}=L$ or $R$. Now let $a_{i}=1$ if $s_{i}=L$ and $a_{i}=-1$ if $s_{i}=R$. In appendix A we show the following 
Theorem 1 The expanded zeta function is a polynomial of degree $n$

$$
1 / \zeta_{\text {top }}(z)=\prod_{p}\left(1-z_{p}^{n}\right)=(1-z) \cdot \sum_{i=0}^{n-1} b_{i} z^{i}
$$

where

$$
\begin{aligned}
& b_{0}=1 \\
& b_{i}=b_{i-1} a_{i} \quad 1 \leq i \leq n-1
\end{aligned} .
$$

If the kneading sequence is infinite (i.e. type b) or c)) the finite sum is replaced by an infinite series.

The function $\sum_{i=0}^{n-1} b_{i} z^{i}$ is called the characteristic polynomial in the literature [10, 15. In ref. [12] it appeared as a characteristic function whose zeros corresponds to eigenvalues of the evolution operator acting on the space of piecewise constant functions.

An important consequence of $(6)$ is that the sequence $\left\{b_{i}\right\}$ has a periodic tail if and only $\mathrm{f}$ the the sequence $\left\{a_{i}\right\}$ has one. We will make use of this observation in section 3 .

\section{A quick journey through parameter space}

The universal sequence of finite kneading sequences is a well studied subject but to make ourselves acquainted with $([6)$ we will discuss some special cases. The letters a) to d) refer to table 1.

a) $\underline{I}_{\lambda}=H^{\infty}(R)$, i.e. the harmonic extension of $R C$, see $[8]$. This is the accumulation point of the first cascade of period doublings. The topological zeta functions is given by $1 / \zeta_{\text {top }}(z)=$ $(1-z) \cdot \prod_{n=0}^{\infty}\left(1-z^{2^{n}}\right)$. The kneading sequence is aperiodic. We will study this function in some detail in the next section. Similar cascades of period doublings accompanies all periodic orbits, or equivalently phrased, a sequence of harmonics follows every finite kneading sequence.

b) $\underline{I}_{\lambda}=R L R^{\infty}$. This is a so called band merging point. The inverse topological zeta function is $1 / \zeta_{\text {top }}(z)=(1-z)\left(1-2 z^{2}\right) /(1+z)$. The kneading sequence is of type $\left.\mathrm{b}\right)$. The preperiodic kneading sequence give rise to the denominator $(1+z)$ above and thus a pole of $1 / \zeta_{\text {top }}(z)$. The two leading zeros $z= \pm 1 / \sqrt{2}$ are of the same size. For the tent map, a slight increase of the control parameter will yield a gap between the leading and next to leading zero and strong mixing. This point, and higher band merging points, has been extensively studied in the literature [12, 13, 14

c) $\underline{I}_{\lambda}=R L C$. The last Sarkovski orbit 10 enters the system, orbits of arbitrary period are allowed. The kneading sequence is $R L C$ and via eqs (5) and (6) we get $1 / \zeta_{\text {top }}(z)=(1-z) \cdot(1-$ $z-z^{2}$ ). This can also be realized as follows (cf. refs. 119 for details). The kneading sequence is $R L C$, so the only forbidden subsequence is $R L L$. All allowed periodic orbits, except $\bar{L}$, can can be built from a alphabet with letters $\underline{R L}$ and $\underline{R}$, following ref. [19] we write this alphabet as $\{\underline{R L}, \underline{R} ; \bar{L}\}$, yielding the topological zeta function $1 / \zeta_{\text {top }}(z)=(1-z)\left(1-z-z^{2}\right)$. The leading zero is the inverse golden mean $z_{0}=(\sqrt{5}-1) / 2$.

d) $\underline{I}_{\lambda}=R L^{\infty}$. The symbolic dynamics is an unrestricted binary one with alphabet $\{\underline{R}, \underline{L}\}$, the zeta function is simply $1 / \zeta_{\text {top }}(z)=1-2 z$. The kneading sequence is of type b) but the associated denominator $(1-z)$ gets canceled by the prefactor in (5).

\section{$3 \quad$ Lacunary zeta functions}

Consider a function $f(z)=\sum c_{i} z^{i}$ defined by a power series. A meromorphic functions has a well defined analytic continuation in the entire complex plane, except at the poles. Functions exhibiting branch point singularities can be continued except on certain imposed cuts. 


\begin{tabular}{|l|l|}
\hline$I(C)$ & $1 / \zeta_{\text {top }}(z) /(1-z)$ \\
\hline$R C$ & \\
$R L R C$ & \\
$R L R R R L R C$ & \\
$a) H^{\infty}(R)$ & $\prod_{n=0}^{\infty}\left(1-z^{2^{n}}\right)$ \\
$R L R R R C$ & \\
$R L R R R R R C$ & \\
$\mathrm{~b}) R L R^{\infty}$ & $\left(1-2 z^{2}\right) /(1+z)$ \\
$R L R R R R R R C$ & \\
$R L R R R R C$ & \\
$R L R R C$ & \\
$R L R R L R C$ & \\
$\mathrm{c}) R L C$ & $\left(1-z-z^{2}\right)$ \\
$R L L R L C$ & \\
$R L L R L R C$ & \\
$R L L R C$ & \\
$R L L R R R C$ & \\
$R L L R R C$ & \\
$R L L R R L C$ & \\
$R L L C$ & \\
$R L L L R L C$ & \\
$R L L L R C$ & \\
$R L L L R R C$ & \\
$R L L L C$ & \\
$R L L L L R C$ & \\
$R L L L L C$ & \\
$R L L L L L C$ & $(1-2 z) /(1-z)$ \\
$\mathrm{d}) R L^{\infty}$ & \\
\hline
\end{tabular}

Table 1: Ordered kneading sequences up to length seven and some longer 
But there is a class of functions that cannot be analytically continued outside a region (typically a disk), the boundary of this region is called a natural boundary. There are some special cases where it is easy to detect the presence of a such a boundary. The classical example is gap series or lacunary series, first studied by Fredholm, Weierstrass and Hadamard. The easiest example is perhaps provided by the series $f(z)=\sum z^{2^{n}}$. The increasing gaps in the sequence of coefficients make the function noncontinuable beyond the circle of convergence $|z|=1$.

Another example is when the coefficients can take on only a finite number of values and the sequence of coefficients does not ultimately become periodic, then the function again has the unit circle as a natural boundary [16]. This result can be directly applied to the expansion of $1 / \zeta_{\text {top }}$, cf eq. (6), for an aperiodic kneading sequence. We can thus get the following theorem for free

Theorem 2 The topological zeta function $1 / \zeta_{\text {top }}$ for unimodal maps has the unit circle as a natural boundary for almost all topological entropies and for the tent map (3), for almost all $\lambda$.

Below we are going to study a specific example of zeta functions exhibiting a natural boundary. The concept of harmonic extension, and the associated cascades of period doublings is a central concept for the description of unimodal maps. This motivates a close study of the function

$$
\Xi(z)=\prod_{n=0}^{\infty}\left(1-z^{2^{n}}\right) .
$$

The expansion of $\Xi(z)$ begins as $\Xi(z)=1-z-z^{2}+z^{3}-z^{4}+z^{5} \ldots$. The radius of convergence is obviously unity. The simple rule governing the expansion will effectively prohibit any periodicity among the coefficients making the unit circle a natural boundary. We will not follow this line of thought, we will instead make a detailed study of the fine structure of this boundary and, as a by product, conclude that the unit circle is opaque.

First we rewrite $\Xi$ as

$$
\Xi(z)=\prod_{n=0}^{\infty}\left(1-\chi_{n}\right),
$$

where the $\chi_{n}$ 's are defined iteratively:

$$
\begin{gathered}
\chi_{n}=\chi_{n-1}^{2} \\
\chi_{0}=z
\end{gathered} .
$$

We write the $\chi_{n}$ on polar form $\chi_{n}=r_{n} \exp \left(2 \pi i x_{n}\right)$ where $r_{n}$ and $x_{n}$ are real numbers and $x_{n}$ is restricted to the unit interval. The iteration rule is

$$
\left\{\begin{array}{c}
r_{n}=r_{n-1}^{2}=r_{0}^{2^{n}} \\
x_{n}=2 x_{n-1} \bmod 1
\end{array} .\right.
$$

Suppose now we choose $r_{0}$ close to, but inside the unit circle $r_{0}=1-\epsilon$. Then $r_{n}$ will be close to unity for $n \leq N \approx \log 2 / \log \epsilon$ and then decrease to zero quickly. By choosing $\epsilon$ we effectively select $N(\epsilon)$ factors of the product representation of $\Xi$ and by decreasing $\epsilon$ we can conclude whether $\Xi(z)$ diverges to $\infty$ or 0 or perhaps converge to a finite value as $z$ approach $\exp (2 \pi i x)$ from within; we thus refer to $z=\exp (2 \pi i x)$ as a pole, zero or a regular point respectively. This reasoning is made rigorous in appendix B.

We will now focus our attention on the second recursion relation in (10) which happens to be the binary shift map, and we will restrict our attention to rational $x_{0}$. Take $x_{0}=p / q$ to be proper reduced fractions, that is $q \in N$ and

$$
p \in \Omega_{q}=\{p ; p \in N, 1 \leq p \leq q,(p, q)=1\},
$$




\begin{tabular}{|l|l|l|l|l|}
\hline$q$ & $\phi(q)$ & $l_{q}$ & $F_{q}(1)$ & \\
\hline 3 & 2 & 2 & 3 & $\infty$ \\
5 & 4 & 4 & 5 & $\infty$ \\
7 & 6 & 3 & 7 & $\infty^{2}$ \\
$9=3^{2}$ & 4 & 4 & 5 & $\infty$ \\
11 & 10 & 10 & 11 & $\infty$ \\
13 & 12 & 12 & 13 & $\infty$ \\
$15=3 \cdot 5$ & 8 & 4 & 1 & $1^{2}$ \\
17 & 16 & 8 & 17 & $0, \infty$ \\
19 & 18 & 18 & 19 & $\infty$ \\
$21=3 \cdot 7$ & 12 & 6 & 1 & $1^{2}$ \\
23 & 22 & 11 & 23 & $\infty^{2}$ \\
$25=5^{2}$ & 20 & 20 & 5 & $\infty$ \\
$27=3^{3}$ & 18 & 18 & 3 & $\infty$ \\
29 & 28 & 28 & 29 & $\infty$ \\
31 & 30 & 5 & 31 & $0^{2}, 1^{2}, \infty^{2}$ \\
$33=3 \cdot 11$ & 20 & 10 & 1 & $0, \infty$ \\
\hline
\end{tabular}

Table 2: Some number theoretic data used to study the structure of the unit circle for the function $\Xi(z)$. Explanations given in section 3 .

where $(p, q)$ denote the greatest common divisor of $q$ and $p$. The number of elements in $\Omega_{q}$ is Euler's function $\phi(q)$. We are thus led to study the following map from $\Omega_{q}$ to itself

$$
p_{n}=2 p_{n-1} \bmod q \text {. }
$$

We will separately consider the three cases.

i/ $q=2^{m}$

ii/ $q=2^{m} q^{\prime}$ where $\left(q^{\prime}, 2\right)=1$

iii/ $(q, 2)=1$

We begin with i/. If we set $r=1^{-}$then only the first $m$ factors of the product (7) are nonzero and all the subsequent will be zero and the corresponding point on the unit circle $z=\exp (2 \pi i x)$ is a zero. These are in a sense trivial zeros and we will eventually discover that they are not the only ones.

Case ii/ reduces to case i/ after $m$ applications of the map.

Now to the more interesting behaviour of the map (12) for case iii/. Below we are going to apply some fundamental results from number theory, they can be found in any standard textbook. The text below is rather compact so in order to digest it could be advisable to verify table 2 , step by step during the reading.

Now $2 \in \Omega_{q}$. It is well known that the set $\Omega_{q}$ is an abelian group with respect to multiplication modulo $q$. This has some important consequences. The map will move points in $\Omega_{q}$ along cycles, because the existence of transients would imply nonexistence of unique inverses. Secondly, if the set $\Omega_{q}$ is divided into several subcycles, all of them has the same length $l_{q}$, and $l_{q}$ is a divisor of $\phi(q)$. The number $l_{q}$ will be the smallest solution to

$$
2^{l_{q}}=1 \bmod q .
$$

Due to Euler's theorem we see that $l_{q}=\phi(q)$ is always a solution but it is not necessarily the smallest. In number theory one says that 2 is a primitive congruence root modulo $q$ if $l_{q}=\phi(q)$, or equivalently, that 2 is a generator of the residue class modulo $q$. 
First, if $q=p$ is a prime then 2 may or may not be a generator. There is no other way than to check it by hand (or look it up in a table). For $p<100$ we can use 2 as a generator for $p=3,5,11,13,19,29,37,53,59,61,67$ and 83 but not for $7,17,23,31,41,43,47,71,73,79,89$ and 97 . If 2 generates the residue class for $q=p$ it also acts as generator for $q=p^{\alpha}$ unless $2^{p-1}=1 \bmod p^{2}$. This is an accident which is very rare if possible, it does not occur for any prime $p<1000$. When building up table 2 we do not have to worry about this complication.

Next suppose $q$ is composite. Than, there does not exist any primitive congruence root and and $l_{q}<\phi(q)$. Indeed, if $q=q_{1} \cdot q_{2}$ and $\left(q_{1}, q_{2}\right)=1$ (and $q_{1}, q_{2} \neq 1$ ) it is fairly easy to show that $l_{q} \leq \phi(q) /\left(\phi\left(q_{1}\right), \phi\left(q_{2}\right)\right) \leq \phi(q) / 2$. We can be more explicit than so: decompose $q$ into prime factors $q=p_{1}^{\alpha_{1}} p_{2}^{\alpha_{2}} \ldots p_{r}^{\alpha_{r}}$ where all $\alpha_{i}>0$. If all residue classes modulo $p_{i}^{\alpha_{i}}$ can be generated by 2 then one can show that $l_{q}=\left\{\phi\left(p_{1}^{\alpha_{1}}\right), \phi\left(p_{2}^{\alpha_{2}}\right), \ldots, \phi\left(p_{r}^{\alpha_{r}}\right)\right\}$, where $\{a, b\}$ stands for least common multiple with obvious generalization for several arguments.

The reader can now verify the third column of table 2 .

The analysis above can tell whether or not the set $\Omega_{q}$ splits up into subcycles under action of the map $p \mapsto 2 p \bmod q$. We can also tell how many subcycles in total having period $n$. The reason is that the binary shift map admits a binary coding of its periodic orbits. There are

$$
M_{n}=\frac{1}{n} \sum_{d \mid n} \mu(n / d) 2^{d}
$$

primitive cycles of length $n, \mu$ is the Möbius function, see e.g. ref. [19] for a derivation. For instance, $M_{3}=2$ so there are two period three cycles, both corresponding to $q=7$, cf. table 2 . $M_{4}=3$ so there are three cycles of period four, corresponding to $q=5, q=9$ and $q=15$.

Next we address the question if a given (sub)cycle $c$ corresponds to a pole, a zero or a regular point of the function $\Xi(z)$. For cycle $c$ we define

$$
\psi_{c}=\prod_{p_{j} \in c}\left(1-e^{2 \pi i p_{j} / q_{c}}\right)
$$

From the previous reasoning it is clear that if $\left|\psi_{c}\right|<1$ then the points $z=\exp \left(2 \pi i p_{j} / q_{c}\right)$ for all $p_{j} \in c$ are zeros of $\Xi(z)$. If $\psi>1$ they are poles and if $\psi=1$ they are regular points. It is not straight forward to express $\psi_{c}$ (without numerical computation) for a given cycle but the product over all cycles with a given $q_{c}=q$

$$
\Psi_{q}=\prod_{q_{c}=q} \psi_{c}=\prod_{1 \leq j<q,(j, q)=1}\left(1-e^{2 \pi i j / q}\right) \equiv F_{q}(1)
$$

is easily expressed. $F_{q}(x)$ are the so called cyclotomic polynomials frequently studied in number theory. We can again use well established results [18] from number theory and state that

If $q$ is a prime power $q=p^{\alpha}$ then $\Psi_{q}=p$, and if $q$ contains two or more distinct prime factors then $\Psi_{q}=1$.

The reader can now verify the fourth column of table 2 .

We were interested in the contribution $\psi_{c}$ from one cycle and if $l_{q}<\phi(q)$ we are only able to compute the product $\Psi_{q}$ of contributions from all cycles corresponding to $q$. In some cases we find that two cycles $c_{1}$ and $c_{2}$ are related by complex conjugation (again a purely number theoretical property): $\Psi_{c_{1}}=\bar{\Psi}_{c_{2}}$ and thus $\left|\Psi_{c_{1}}\right|=\left|\Psi_{c_{2}}\right|$. This is the case for e.g. $q=7$. When there is no such symmetry there seems to preference to produce some $c$ such that $\left|\Psi_{c}\right|<1$ leading to zeros of $\Xi(z)$ that are surely non trivial.

In the fifth column we indicate whether the different subcycles corresponds to zeros, poles or regular points. Many entries required explicit calculations.

As a special case we can state the following 
Theorem 3 The sets of zeros and poles of $\Xi(z)$ are dense on the unit circle.

To show that the zeros (poles) are dense it suffice to consider points $z=\exp (2 \pi i p / q)$ where $q$ is a power of two (three), $q=2^{m}\left(q=3^{m}\right)$. The function $\Xi(z)$ can thus not be continued outside the unit circle.

We will only speculate what happens for irrational $x$, that is points that are not periodic points of the binary shift map. We know that this map is ergodic and the invariant density is uniform. Therefore, the fact the the following integral is zero

$$
\int_{0}^{1} \log \left(1-e^{2 \pi i x}\right) d x=0,
$$

leads us to believe that irrational $x$ corresponds to regular points of $\Xi(z)$.

\section{Concluding remarks}

As we remarked before, our findings concerning the topological zeta functions can be carried over directly to general zeta function

$$
1 / \zeta(\beta, z)=\prod_{p}\left(1-\frac{z^{n_{p}}}{\left|\Lambda_{p}\right|^{1-\beta}}\right),
$$

only for piecewise linear maps when $0 \leq \beta<1$. However for expanding maps, the topological zeta function will, after proper rescaling of $z$, a la eq. (ब), approximate the exact expansion of the zeta function and correspond to the fundamental part in a cycle expansion 19. It is not clear in what sense the curvature corrections of [19] are small in this case but it is natural to expect the natural boundary to prevail. However, the methods used in this paper do not immediately apply to the non-linear case so we leave that as a speculation.

The reasoning above suggests that one could expect a boundary along

$$
|z|=\left\langle\left|f^{\prime}\right|^{1-\beta}\right\rangle
$$

when there is no finite Markov partition. $\langle$.$\rangle is some suitable defined average, presumably geo-$ metric.

In ref. 21] it is shown that for piecewise monotone maps $1 / \zeta(z)$ is holomorphic inside

$$
z<\lim _{n \rightarrow \infty} \sup \left(\prod_{i=1}^{n}\left|f^{\prime}\left(x_{i}\right)\right|\right)^{\frac{1-\beta}{n}}
$$

where $x_{i+1}=f\left(x_{i}\right)$ and the supremum is taken over the initial point $x_{1}$. The only requirement is that the weight, in this case $1 /\left|f^{\prime}\right|^{1-\beta}$ is of finite variation. (For more recent development on this subject see [22, 23, 24].) If there exists a finite Markov partition we know that this holomorphic disk may be limited by a pole of $1 / \zeta(z)$. What we suggest is that in the generic situation with no finite Markov partition this guaranteed holomorphic disk has encountered a natural boundary.

The convergence of cycle expansion depends on the distance between the zero to be determined and the nearest singularity of $1 / \zeta(z)$, zeros close to a natural boundary will be difficult to locate accurately. When looking for zeros of the truncated power series expansion of $1 / \zeta(z)$ one will typically find that zeros accumulate along the boundary as the truncation length is increased 25. The situation would hardly be improved by applying some continued fraction expansion of the zeta function. Such an approach could be a good idea if the (inverse) zeta function is 
meromorphic, we have encountered such a situation for the preperiodic case. But the presence of a natural boundary would presumably lead to accumulation of zeros and poles along the boundary of the successive rational approximations of the zeta function.

I would like to thank Viviane Baladi and Predrag Cvitanović for discussions and remarks. This work was supported by the Swedish Natural Science Research Council (NFR) under contract no. F-FU 06420-303.

\section{Appendix A}

In this appendix we will prove Theorem 1, that is, we will show that the expansion coefficients $\left\{b_{i}\right\}$ in

$$
1 / \zeta_{\text {top }}=\prod_{p}\left(1-z_{p}^{n}\right)=(1-z) \cdot \sum_{i=0}^{n-1} b_{i} z^{i}
$$

are given by

$$
\begin{aligned}
& b_{0}=1 \\
& b_{i}=b_{i-1} a_{i}=\prod_{j=1}^{i} a_{i} \quad 1 \leq i \leq n-1,
\end{aligned}
$$

where the coefficients $\left\{a_{i}\right\}$ are obtained from the finite kneading sequence $\underline{I}_{\lambda}=P C$, of length $n$, where $P=s_{1} s_{2} \ldots s_{n-1}$, according to the rule that $a_{i}=1$ if $s_{i}=L$ and $a_{i}=-1$ if $s_{i}=R$. The result follows directly from the results of Milnor and Thurston [20]. We first recall some expressions from [20]. Let $J$ be an subinterval of the unit interval, and $f^{n}$ the n'th iterate of the map. Then one defines

$$
\theta\left(x, J, f^{m}(x)\right)=\left\{\begin{array}{cc}
1 & f^{m}(x) \in J \text { and } \frac{d f^{m}(x)}{d x}>0 \\
-1 & f^{m}(x) \in J \text { and } \frac{d f^{m}(x)}{d x}<0 \\
0 & f^{m}(x) \notin J
\end{array} .\right.
$$

We use the convention that $f^{0}(x)=x$. Let the unit interval be divided into $I_{1}$ and $I_{2}$ by the critical point $c$ (corresponding to the symbol $C$ ). For a map with one turning point, the kneading matrix is a $1 \times 2$ matrix $\left(K_{1}, K_{2}\right)$ where

$$
K_{i}=\sum_{m=0}^{\infty}\left(\theta\left(c^{-}, I_{i}, f^{m}\right)-\theta\left(c^{+}, I_{i}, f^{m}\right)\right) z^{m} .
$$

Perturbation of the critical point as initial value leads to the sequence $C^{ \pm} P \overline{C^{+} P}$ of $P$ contains an even number of $R$ 's and $C^{ \pm} P \overline{C^{-} P}$ if it contains an odd. We also note that if $1 \leq m \leq n-1$

$$
\begin{gathered}
\frac{d f^{m}(c+)}{d x}=-b_{m-1} \\
\frac{d f^{m}(c-)}{d x}=+b_{m-1} .
\end{gathered}
$$


A short calculation now yields

$$
K_{1}+K_{2}=\frac{\sum_{m=1}^{n} 2 b_{m-1} z^{m}}{1-z^{n}}=2 z \frac{\sum_{m=0}^{n-1} b_{m} z^{m}}{1-z^{n}} .
$$

The matrix elements $K_{1}$ and $K_{2}$ are related by

$$
(1-z) K_{1}+(1+z) K_{2}=0
$$

We can now express the kneading determinant

$$
D(z)=\frac{K_{1}}{1+z}=\frac{\left(K_{1}+K_{2}\right)(1+z)}{2 z(1+z)}=\frac{\sum_{m=0}^{n-1} b_{m} z^{m}}{1-z^{n}} .
$$

This determinant is related to the inverse topological zeta function $1 / \zeta_{\text {top }}$ according to 20]

$$
1 / \zeta_{\text {top }}=D(z)\left(1-z^{n}\right)(1-z)=(1-z) \sum_{m=0}^{n-1} b_{m} z^{m}
$$

as claimed.

If the kneading sequence is infinite (i.e. preperiodic or aperiodic) then the factor $\left(1-z^{n}\right)$ disappears from eq. (29) (obvious) and from eq. (30) (according to ref. [20]) and the only alteration of the final result is that $n$ is replaced by $\infty$.

\section{Appendix B}

Consider the function

$$
\Xi\left(z=r_{0} e^{2 \pi i x_{0}}\right)=\prod_{n=0}^{\infty}\left(1-r_{0}^{2^{n}} e^{2 \pi i x_{n}}\right) .
$$

We are interested in the behaviour of $\Xi$ when $r_{0} \rightarrow 1^{-} . x_{0}$ is kept fixed and choosen so that the sequence $\left\{x_{n}\right\}$ is periodic: $x_{n+m}=x_{n}$. We rewrite the previous equation as

$$
\Xi\left(z=r_{0} e^{2 \pi i x_{0}}\right)=\prod_{k=0}^{\infty} f\left(r_{0}^{2^{m k}}\right)
$$

where

$$
f(r)=\prod_{l=0}^{m-1}\left(1-r^{2^{l}} e^{2 \pi i x_{n}}\right) .
$$

First we consider the case $|f(1)|>1$, and we will show that $|\Xi| \rightarrow \infty$ as $r_{0} \rightarrow 1^{-}$. Obviously $f(r)$ is an entire analytic function and in particular a continous function so that there exist some $\tilde{r}$ such that $|f(r)|>C>1$ for all $1>r \geq \tilde{r}$. We split up the product

$$
\Xi\left(r_{0} e^{2 \pi i x_{0}}\right)=\prod_{k=0}^{\tilde{k}-1} f\left(r_{0}^{2^{m k}}\right) \cdot \prod_{k=\tilde{k}}^{\infty} f\left(r_{0}^{2^{m k}}\right) \equiv D(\tilde{r}) \cdot \prod_{k=0}^{\tilde{k}-1} f\left(r_{0}^{2^{m k}}\right) .
$$

We choose $r_{0}^{2^{m \tilde{k}}}=\tilde{r}$ so that $D(\tilde{r})$ is a constant

$$
D(\tilde{r})=\prod_{k=\tilde{k}}^{\infty} f\left(r_{0}^{2^{m k}}\right)=\prod_{k=0}^{\infty} f\left(\tilde{r}^{2^{m k}}\right),
$$


depending only on our choice of $\tilde{r}$. It is finite because $\tilde{r}<1$ and $\Xi(z)$ converges inside the unit circle. We now have

$$
|\Xi|>D(\tilde{r}) \cdot C^{\tilde{k}}
$$

which can be made arbitrary large by choosing $\tilde{k}$ sufficiently large and thus $r$ sufficiently close to unity.

The case when $|f(1)|<1$ is worked out in complete analogy it is is shown that $|\Xi| \rightarrow 0$ as $r_{0} \rightarrow 1^{-}$.

The case $f(1)=1$ requires just a little more work. First we rewrite eq. (34)

$$
\Xi\left(r_{0} e^{2 \pi i x_{0}}\right)=D(\tilde{r}) \cdot \prod_{k=0}^{\tilde{k}-1} f\left(\tilde{r}^{2^{m(k-\tilde{k})}}\right)=D(\tilde{r}) \cdot \prod_{k=1}^{\tilde{k}} f\left(\tilde{r}^{2^{-m k}}\right) .
$$

We want to show that the product converges when $\tilde{k} \rightarrow \infty$, and thus $r_{0} \rightarrow 1^{-}$. We appeal again to the analyticity of $f(r)$. Since $f(1)=1$ there exist a constant $E$ such that

$$
|f(r)-1|<E(1-r) \quad \tilde{r} \leq r<1 .
$$

The product (37) converges if

$$
\sum_{k=0}^{\infty}\left|f\left(\tilde{r}^{2^{-m k}}\right)-1\right|<\infty
$$

Using (38) we get the condition

$$
\sum_{k=0}^{\infty}\left|f\left(\tilde{r}^{-m k}\right)-1\right|<E \sum_{k=0}^{\infty}\left(1-\tilde{r}^{2^{-m k}}\right)
$$

which converges since

$$
\left(1-\tilde{r}^{2^{-m k}}\right)=O\left(-\log \tilde{r} 2^{-m k}\right) \quad k \rightarrow \infty
$$




\section{References}

[1] D. Ruelle, Inv. Math. 34 (1976) 231.

[2] H. H. Rugh, Nonlinearity 5, 1237, (1992).

[3] S. Isola, Dynamical zeta functions and correlation functions for non-uniformly hyperbolic transformations, Preprint, Bologna (1995).

[4] P. Dahlqvist, Nonlinearity 8,11 (1995).

[5] P. Dahlqvist, Do zeta functions for intermittent maps have branchpoints, preprint, Stockholm (1996).

[6] P. Dahlqvist, Lyapunov exponents and anomalous diffusion of a Lorentz gas with infinite horizon using approximate zeta functions, J. Stat. Phys. to appear.

[7] P. Dahlqvist and R. Artuso, On the decay of correlations in Sinai billiards with infinite horizon, submitted to Phys. Lett. A .

[8] N. Metropolis, M. L. Stein and P. R. Stein, J. Combinatorial Theory 15 (1973) 25.

[9] J. Guckenheimer, Inv. Math. 39 (1977) 165.

[10] P. Collet, J.P. Eckmann Iterated maps on the unit interval as dynamical systems (Birkhäuser, Boston 1980).

[11] C. Beck, F. Schlögl, Thermodynamics of chaotic systems, Cambridge Nonlinear Science Series 4, Cambridge (1993).

[12] M. Dörfle, J. Stat. Phys. 40, (1985) 93.

[13] H. Shigematsu, H. Mori, T. Yoshida and H. Okamuto, J. Stat. Phys. 30 (1983) 649.

[14] T. Yoshida, H. Mori and H. Shigematsu, , J. Stat. Phys. 31 (1983) 279.

[15] L. Jonker, Proc. London Math. Soc. 39 (1979) 428.

[16] E. Hille, Analytic function theory II (1959).

[17] R. Artuso, E. Aurell and P. Cvitanović, Nonlinearity 3, 325 and 361, (1990).

[18] R. Sivaramakrishnan, Classical theory of aritmetic functions, Pure and applied mathematics no. 126, Marcel Dekker, New York (1989).

[19] R. Artuso, E. Aurell and P. Cvitanović, Nonlinearity 3, 325 and 361, (1990).

[20] J. Milnor and W. Thurston, in Lecture notes in Mathematics, No. 1342, J. C. Alexander, ed. (1988) 465.

[21] V. Baladi and G. Keller, Comm. Math. Phys. 127 (1990) 459.

[22] V. Baladi and D. Ruelle, Ergod. Th. Dynam. Sys. 14, (1994) 621.

[23] V. Baladi and D. Ruelle, Invent. Math. 123, (1996) 553.

[24] V. Baladi, J. Func. Anal. 128 (1995) 226

[25] K. T. Hansen, thesis, Oslo (1900). 